\title{
Investigation of the energy-averaged double transition density of isoscalar monopole excitations in medium-heavy mass spherical nuclei
}

\author{
M.L. Gorelik ${ }^{1,2}$, S. Shlomo ${ }^{1,2}$, B.A. Tulupov ${ }^{1,3}$, and M.H. Urin ${ }^{1}$ \\ ${ }^{1}$ National Research Nuclear University «MEPhI», Moscow, 115409 Russia \\ ${ }^{2}$ Cyclotron Institute, Texas A\&M University, College Station, Texas 77843, USA \\ ${ }^{3}$ Institute for Nuclear Research, RAS, Moscow, 117312 Russia
}

The particle-hole dispersive optical model, developed recently, is applied to study properties of high-energy isoscalar monopole excitations in medium-heavy mass spherical nuclei. The energy-averaged strength functions of the isoscalar giant monopole resonance and its overtone in ${ }^{208} \mathrm{~Pb}$ are analyzed. In particular, we analyze the energy-averaged isoscalar monopole double transition density, the key quantity in the description of the hadron-nucleus inelastic scattering, and studied the validity of the factorization approximation using semi classical and microscopic one body transition densities, respectively, in calculating the cross sections for the excitation of isoscalar giant resonances by inelastic alpha scattering.

PACS number(s): 21.60.Jz, 24.30.Cz, 21.60.Ev, 24.10.Nz

\section{INTRODUCTION}

The study of properties of collective states in nuclei provides information on the bulk properties of nuclear matter. In particular, the interest in experimental and theoretical studies of high-energy particle-hole-type isoscalar monopole (ISM) excitations in medium-heavy mass nuclei is mainly due to the possibility of determining the nuclear matter incompressibility coefficient, a fundamental physical quantity essential for astrophysics and nuclear physics. The value of this coefficient depends on the mean energy of the strength distribution, corresponding to the ISM external field $r^{2} Y_{00}$ (in other words, on the energy of the isoscalar giant monopole resonance (ISGMR)) [1]. To deduce this strength distribution from experimental data of $\left(\alpha, \alpha^{\prime}\right)$ inelastic scattering cross sections at small angles, it is usually assumed that the ISM strength is concentrated in the vicinity of the ISGMR and the properly normalized classical collective model transition density of the ISGMR can be used within the folding model distorted wave Born approximation (FM-DWBA) (see. e.g., Ref. [2] for details). It is important to point out that the classical collective model one-body transition density is independent of the excitation energy. In Ref. [3], a microscopic evaluation of the $\left(\alpha, \alpha^{\prime}\right)$-scattering cross sections were carried out within the FM-DWBA using the Hartree-Fock (HF) ground state density and the one-body transition densities obtained from the HF-based random-phase-approximation (RPA) calculations. A comparison with results obtained with the classical collective model transition density was also made in Ref. [3]. 
In this work we apply the newly developed particle-hole (p-h) dispersive optical model (PHDOM) to study properties of high-energy isoscalar monopole excitations in ${ }^{208} \mathrm{~Pb}$. $\mathrm{A}$ microscopically-based (phenomenological) description of the spreading effect on properties of high-energy particle-hole-type excitations is the specific feature of the PHDOM. The model is formulated in terms of the corresponding energy-averaged Green functions. (The Green function method for description of simple modes of nuclear excitations has been first exploited by Migdal [4]). The basic quantity of the PHDOM is the "free" p-h Green function, corresponding to the model of independently damping quasiparticles. The use of the statistical assumption (which means that after energy averaging the different particle-hole states are "decaying" into manyquasiparticle configurations independently of one another) allows one to get the expression for the mentioned Green function in a closed form [5,6]. Being a direct generalization of the corresponding discrete-RPA free $\mathrm{p}$ - $\mathrm{h}$ propagator, this expression contains the (phenomenological) imaginary and real parts of the strength of an energy-averaged specific p-h interaction ( $\mathrm{p}$-h self-energy term) responsible for the spreading effect. The imaginary part determines the real one via the proper dispersive relationship, which follows from the spectral expansion of the $2 \mathrm{p}-2 \mathrm{~h}$ Green function. An attempt to take explicitly within the model the singleparticle continuum into account (i.e., to get a generalization of the continuum-RPA [7]) has been realized in Refs. [5,6] with the use of an approximate spectral expansion for the Green function of the single-particle Shrodinger equation, which contains the imaginary and dispersive real additions to the mean field. Appearance of such a Green function allows one to call the model as the $\mathrm{p}$-h dispersive optical model. It should be stressed, however, that the above-mentioned imaginary addition to the mean field is not related to the imaginary part of the potential widely used for description of the nucleon-nucleus scattering within the single-particle optical model. The first intensive implementation of the PHDOM has been realized in Ref. [8] in applying to description of simplest photonuclear reactions. We note that a simple version of the mean field and particle-hole interaction are used within the PHDOM in order to take into account the Landau damping, single-particle continuum and the spreading effect, simultaneously.

We emphasize that in a microscopic approach, the input quantity for the analysis of the $\left(\alpha, \alpha^{\prime}\right)$-reaction cross section should be the energy-averaged double transition density (i.e. the energy-averaged product of energy dependent transition densities taken in different points). In a wide excitation-energy interval involving the ISGMR and its overtone, ISGMR2, this quantity is expected to be different from the product of the classical collective model transition densities [2], which is independent of excitation energy, or the product of microscopic transition densities [3], due to proper treatment of the shell structure of nuclei (i.e. the Landau damping) and also the spreading effect. The PHDOM allows one to describe the energy-averaged ISM double transition density at arbitrary (but high-enough) excitation energy and, in particular, to trace the change of this quantity from the ISGMR to ISGMR2.

In Ref. [9] we have presented the first evaluation of the energy-averaged double $p-h$ transition density within the PHDOM with very limited results. In this work we present results of extended investigation of the energy-averaged double transition density. The calculations are performed for ISM excitations in ${ }^{208} \mathrm{~Pb}$. A wide excitation-energy interval is considered which 
includes the ISGMR and ISGMR2. The fractions of the energy-weighted sum-rule (EWSR) associated with the strength functions of these resonances, i.e. the energy-weighted strength functions divided by the corresponding EWSR, are analyzed. We also consider single p-h transition density obtained by various projections of the double $\mathrm{p}-\mathrm{h}$ transition density, as well as the classical collective models transition densities, and investigate their applicability. This is done by considering the excitation cross sections with the Born approximation and comparing with the results obtained from the double $\mathrm{p}-\mathrm{h}$ transition density. We add that it is important to carry out this test on the accuracy of the experimental analysis of cross sections before a reliable comparison between experimental data with theoretical prediction obtained using energy density functional.

\section{FORMALISM}

As mentioned in Introduction, within the PHDOM, the continuum-RPA is extended to take into account the spreading effect phenomenologically with averaging over the energy. The basic ingredient of the model is the energy-averaged $\mathrm{p}$-h Green function (or the effective $\mathrm{p}-\mathrm{h}$ propagator), $A^{\alpha \beta}$, where $\alpha, \beta=n, p$ are the isobaric indexes. The "free" $\mathrm{p}$-h propagator, which corresponds to the model of independent and damping quasiparticles, is diagonal in these indexes: $A_{0}^{\alpha, \beta}=A_{0}^{\alpha} \delta_{\alpha \beta}$. The properties of isoscalar excitations are described by a propagator obtained as a proper sum: $A=\sum_{\alpha, \beta=n, p} A^{\alpha \beta}$, with $A_{0}=\sum_{\alpha=n, p} A_{0}^{\alpha}$. The ISM radial component of this propagator, $\left(r^{2} r^{\prime 2}\right)^{-1} A\left(r, r^{\prime}, \omega\right)$, determines the energy-averaged ISM radial double transition density

$$
-\frac{1}{\pi} \operatorname{Im} A\left(r, r^{\prime}, \omega\right)=\left\langle\rho(r, \omega) \rho\left(r^{\prime}, \omega\right)\right\rangle=\rho\left(r, r^{\prime}, \omega\right) .
$$

In Eq. (1), $\omega$ is the excitation energy $\left(\omega>B_{\alpha}, B_{\alpha}\right.$ is the nucleon separation energy), $\rho(r, \omega)=\sum_{\alpha=n, p} \rho^{\alpha}(r, \omega)$ is the radial one-dimensional transition density and the brackets $\langle\ldots\rangle$ denote averaging over the energy. We point out that the three-dimensional ISM transition density is determined as $\rho(r, \omega)=r^{-2} \rho(r, \omega) Y_{00}(n)$ so that the corresponding ISM double transition density is given by:

$$
\rho\left(r, r^{\prime}, \omega\right)=\frac{1}{4 \pi}\left(r r^{\prime}\right)^{-2} \rho\left(r, r^{\prime}, \omega\right) .
$$

In accordance with Eq. (1), the energy-averaged strength function $S_{V_{0}}(\omega)$, associated with the ISM external s-p field $V_{0}(\dot{r})=V_{0}(r) Y_{00}(\dot{h})$, is determined by the averaged effective p-h propagator:

$$
S_{V_{0}}(\omega)=-\frac{1}{\pi} \operatorname{Im} \int V_{0}(r) A\left(r, r^{\prime}, \omega\right) V_{0}\left(r^{\prime}\right) d r d r^{\prime}=-\frac{1}{\pi} \operatorname{Im} \int V_{0}(r) A_{0}\left(r, r^{\prime}, \omega\right) V\left(r^{\prime}, \omega\right) d r d r^{\prime} .
$$

We add that within the PHDOM, there is an equivalent and more simple method for evaluation of the energy-averaged strength function by using the effective field $V(r, \omega)$, which corresponds to an external s-p field $V_{0}(r)$. The effective field, determined by the equation 


$$
\int A\left(r, r^{\prime}, \omega\right) V_{0}\left(r^{\prime}\right) d r^{\prime}=\int A_{0}\left(r, r^{\prime}, \omega\right) V\left(r^{\prime}, \omega\right) d r^{\prime},
$$

satisfies a more simple equation than the below-given equation for $A\left(r, r^{\prime}, \omega\right)$ (see, e.g., Refs. [10-12]). However, this method cannot be used for the evaluation of the energy-averaged double transition density $[5,6]$. To describe the properties of the ISGMR and ISGMR2, it is convenient to choose for the radial external fields the forms

$$
V_{0,1}(r)=r^{2}, \quad V_{0,2}(r)=r^{4}-\eta r^{2},
$$

respectively. In the present work, unlike the approaches of Refs. [11,13], we find the parameter $\eta$ by minimizing the quantity $x_{2}(\eta)=\int y_{2}(\omega) d \omega, y_{2}(\omega)=\omega S_{V_{0,2}}(\omega) /(E W S R)_{V_{0,2}}$, where the integration is performed over the ISGMR region.

We point out that the Bethe-Goldstone equation for the energy-averaged effective $\mathrm{p}-\mathrm{h}$ propagator is given in Refs. [5,6] in a rather general form. This equation is given below in the form which is directly used for the evaluation of the ISM basic quantity $((N-Z)<<A)$ :

$$
A\left(r, r^{\prime}, \omega\right)=A_{0}\left(r, r^{\prime}, \omega\right)+\int A_{0}\left(r, r_{1}, \omega\right) F\left(r_{1}\right) A\left(r_{1}, r^{\prime}, \omega\right) r_{1}^{-2} d r_{1} .
$$

In (6), $F(r)$ is the strength of the isoscalar part of the Landau-Migdal p-h interaction [4]: $F\left(\dot{r}_{1}, \dot{r}_{2}\right) \rightarrow F\left(r_{1}\right) \delta\left(\dot{r}_{1}-\dot{r}_{2}\right)$. In accordance with Refs. [5,6,8], the expression for the "free" p-h propagator $A_{0}\left(r, r^{\prime}, \omega\right)$ can be presented in the form (the isobaric index $\alpha$ is omitted for brevity):

$$
\begin{aligned}
A_{0}\left(r, r^{\prime}, \omega\right) & =\sum_{\mu,(\lambda)} n_{\mu} t_{(\lambda)(\mu)}^{2} \chi_{\mu}(r) \chi_{\mu}\left(r^{\prime}\right) g_{(\lambda)}\left(r, r^{\prime}, \varepsilon_{\mu}+\omega\right)+\sum_{\lambda,(\mu)} n_{\lambda} t_{(\mu)(\lambda)}^{2} \chi_{\lambda}(r) \chi_{\lambda}\left(r^{\prime}\right) g_{(\mu)}\left(r, r^{\prime}, \varepsilon_{\lambda}-\omega\right)+ \\
& +2 \sum_{\mu, \lambda} n_{\mu} n_{\lambda} t_{(\mu)(\lambda)}^{2} \chi_{\mu}(r) \chi_{\mu}\left(r^{\prime}\right) \chi_{\lambda}(r) \chi_{\lambda}\left(r^{\prime}\right) \frac{(i W(\omega)-P(\omega)) f_{\mu} f_{\lambda}}{\left(\varepsilon_{\lambda}-\varepsilon_{\mu}-\omega\right)^{2}+(i W(\omega)-P(\omega))^{2} f_{\mu}^{2} f_{\lambda}^{2}} .
\end{aligned}
$$

In (7), $t_{(\lambda)(\mu)}^{2}=\frac{2 j_{\mu}+1}{4 \pi} \delta_{(\lambda)(\mu)}, \quad n_{\alpha}$ is the occupation factor for the s-p level $\mu$, which is characterized by the energy $\varepsilon_{\mu}$ and by the set of quantum numbers of the total and orbital momenta $(\mu) \equiv\left(j_{\mu}, l_{\mu}\right) ; r^{-1} \chi_{\mu}(r)$ is the bound state radial wave function, $f_{\mu}=\int f_{W S}(r) \chi_{\mu}^{2}(r) d r$ and $f_{W S}(r)$ is the well-known Woods-Saxon function. Being the imaginary and real parts of the strength of an energy-averaged specific $\mathrm{p}$-h interaction responsible for the spreading effect, the phenomenological quantities $W(\omega)$ and $P(\omega)$ determine the optical-model-like addition to the nuclear mean field used in the calculations of the optical-model radial Green functions $\left(r r^{\prime}\right)^{-1} g\left(r, r^{\prime}, \omega\right)$ :

$$
\begin{aligned}
& \left.\left(h_{0,(\lambda)}(r)-\left\{\varepsilon_{\mu}+\omega+(i W(\omega)-P(\omega)) f_{\mu} f_{W S}(r)\right\}\right)\right)_{(\lambda)}\left(r, r^{\prime}, \varepsilon_{\mu}+\omega\right)=-\delta\left(r-r^{\prime}\right), \\
& \left.\left(h_{0,(\mu)}(r)-\left\{\varepsilon_{\lambda}-\omega+(i W(\omega)-P(\omega)) f_{\lambda} f_{W S}(r)\right\}\right)\right)_{(\mu)}\left(r, r^{\prime}, \varepsilon_{\lambda}-\omega\right)=-\delta\left(r-r^{\prime}\right) .
\end{aligned}
$$

Here, $(\lambda)=(\mu)$ and $h_{0}(r)$ are the radial parts of the s-p Hamiltonian (including the spin-orbit and centrifugal terms).

Completing the brief description of the basic PHDOM equations, we note that there is a weak violation of the model unitarity. The violation is caused by the energy dependence of the effective optical-model potential and also by the use of the approximate spectral expansion for 
the optical-model Green functions of Eqs. (8) and (9). Methods for restoration of the model unitarity are under consideration. Some preliminary results are presented in Ref. [14].

It is common to use the energy-averaged classical double transition densities of the ISGMR and ISGMR2, corresponding to single-level resonances, and presented them in the factorized form:

$$
\begin{gathered}
\rho_{c, i}^{L}\left(r, r^{\prime}, \omega\right)=\rho_{c, i}^{L}(r, \omega) \rho_{c, i}^{L}\left(r^{\prime}, \omega\right)=L_{i}(\omega) \rho_{c, i}(r) \rho_{c, i}\left(r^{\prime}\right), \\
L_{i}(\omega)=\frac{\Gamma_{i}}{2 \pi}\left(\left(\omega-\omega_{i}\right)^{2}+\frac{1}{4} \Gamma_{i}^{2}\right)^{-1} .
\end{gathered}
$$

Here, $\omega_{i}$ and $\Gamma_{i}$ are the resonance energy and total width, respectively. The classical collective model transition densities for the ISGMR (i=1) and ISGMR2 (i=2), normalized to the corresponding energy-weighted sum rules, are given by [13]:

$$
\begin{gathered}
\rho_{c, 1}(r)=C_{1} r^{2} \sqrt{4 \pi}\left(3+r \frac{d}{d r}\right) n(r), C_{1}^{2}=\frac{\mathrm{h}^{2}}{2 m A \omega_{1}\left\langle r^{2}\right\rangle}, \\
\rho_{c, 2}(r)=C_{2} r^{2} \sqrt{4 \pi}\left(10 r^{2}-3 \eta_{c}+r\left(2 r^{2}-\eta_{c}\right) \frac{d}{d r}\right) n(r), \\
C_{2}^{2}=\frac{\mathrm{h}^{2}}{2 m A \omega_{2}\left(4\left\langle r^{6}\right\rangle-4 \eta_{c}\left\langle r^{4}\right\rangle+\eta_{c}^{2}\left\langle r^{2}\right\rangle\right)}, \eta_{c}=\frac{2\left\langle r^{4}\right\rangle}{\left\langle r^{2}\right\rangle},
\end{gathered}
$$

where $n(r)=\sum_{\alpha} n^{\alpha}(r)$ is the ground state matter density, $m$ is the nucleon mass, the brackets $\langle\ldots\rangle$ mean here averaging over the mentioned density. We point out that in the present work we do not adopt the strength of the classical transition densities defined via the Lorentzian $L_{i}(\omega)$, Eq. (10). To conform with the widely-used method of analyzing the experimental excitation cross sections, we instead normalize the classical transition density $\rho_{c, i}^{\Lambda}(r, \omega)=\Lambda_{i}^{1 / 2}(\omega) \rho_{c, i}(r)$ by imposing the condition of reproducing the microscopic energy-weighted strength functions, i.e. imposing the condition

$$
\left(\int \rho_{c, i}^{\Lambda}(r, \omega) V_{0, i}(r) d r\right)^{2}=\int V_{0, i}(r) \rho\left(r, r^{\prime}, \omega\right) V_{0, i}\left(r^{\prime}\right) d r d r^{\prime},
$$

using Eqs. (5), (11) and (12) for the external fields, the classical transition densities of the ISGMR and ISGMR2. As follows from Eqs. (1), (3) and (13), $\Lambda_{i}(\omega)=S_{V_{0, i}}(\omega) /\left(\int \rho_{c, i}(r) V_{0, i}(r) d r\right)^{2}=\omega_{i} S_{V_{0, i}}(\omega) /(E W S R)_{V_{0, i}}$. The resulting microscopically corrected classical double transition densities, $\rho_{c, i}^{\Lambda}\left(r, r^{\prime}, \omega\right)$, will be compared with that obtained in our microscopic calculations. This comparison permits also to determine the sign of $\rho_{c, i}^{\Lambda}\left(r, r^{\prime}, \omega\right)$ which can not be defined from Eq. (13).

We also study possibilities of an appropriate factorization of the ISM double-transitiondensity radial dependence in a wide excitation-energy interval. For this purpose we define the projected transition density (adopted in Ref. [3]),

$$
\rho_{V_{0}}(r, \omega)=\int \rho\left(r, r^{\prime}, \omega\right) V_{0}\left(r^{\prime}\right) d r^{\prime} / S_{V_{0}}^{1 / 2}(\omega),
$$

which can be considered as the transition density of a given ISM giant resonance (ISGMR or its overtone). This transition density, employed in Refs. [3,10-13] as the energy-dependent 
transition density of the corresponding giant resonance, fulfils the condition that the strength function is obtained by

$$
S_{V_{0}}(\omega)=\left(\int \rho_{V_{0}}(r, \omega) V_{0}(r) d r\right)^{2}
$$

The projected transition density can be expressed also in terms of the effective field [10-12]:

$$
\rho_{V_{0}}(r, \omega)=-\frac{1}{\pi} \operatorname{Im} \frac{V(r, \omega)}{F(r) S_{V_{0}}^{1 / 2}(\omega)} .
$$

The distorted wave Born approximation (DWBA) is widely used in the analysis of measured cross sections of scattering probes. The energy-averaged DWBA differential cross section for the excitation of a giant resonance by inelastic $\alpha$-scattering is given by

$$
\left\langle\frac{d \sigma^{D W B A}}{d \Omega_{\alpha^{\prime}} d E_{\alpha^{\prime}}}\right\rangle=\left(\frac{\mu}{2 \pi \mathrm{h}^{2}}\right)^{2} \frac{k_{\alpha^{\prime}}}{k_{\alpha}}\left\langle\sum_{s}\left|T_{\alpha^{\prime} s, \alpha 0}\right|^{2} \delta\left(E_{s}-E_{0}-\omega\right)\right\rangle,
$$

where $\mu$ is the $\alpha$-particle reduced mass and $\mathrm{h} k_{\alpha}=\sqrt{2 \mu E_{\alpha}}$ and $\mathrm{h} k_{\alpha^{\prime}}=\sqrt{2 \mu\left(E_{\alpha}-\omega\right)}$ are the initial and final momenta of the $\alpha$-nucleus relative motion, respectively. The transition matrix element is given by

$$
\left.T_{\alpha^{\prime} s, \alpha 0}=\left(\varphi_{\alpha^{\prime}}^{(-)} \stackrel{r}{r} r_{\alpha}\right) \psi_{s}(\stackrel{r}{r})\left|V_{\alpha A}\right| \varphi_{\alpha}^{(+)}(\stackrel{r}{r}) \psi_{\alpha}(\stackrel{r}{r})\right) .
$$

Here, $V_{\alpha A}=\sum_{i} V\left(r_{\alpha}^{r}-r_{r}^{r}\right)$ is the $\alpha$-nucleus interaction, where $V$ is the $\alpha$-nucleon interaction and $r_{\alpha}$ and $r_{i}$ are the coordinates of the $\alpha$-particle and the nucleon, respectively. In Eq. (18), $\psi_{0}$ and $\psi_{s}$ are the wave functions of the ground and excited states of the nucleus, and $\varphi_{\alpha}^{(+)}, \varphi_{\alpha^{\prime}}^{(-)}$are the corresponding distorted wave functions of the $\alpha$-nucleus relative motion. We emphasize that the energy-averaged cross-section is obtained from Eq. (17) by using the energy-averaged transition strength function. Therefore, the input to the DWBA calculations should be the energy-averaged double transition density $\rho\left(r, r^{\prime}, \omega\right)$ of Eq. (1) (see the spectral expansion of $A\left(r, r^{\prime}, \omega\right)$, e.g., in Refs. [5,6]).

In the experimental analysis of inelastic $\left(\alpha, \alpha^{\prime}\right)$-scattering cross sections at small angles the folding model (FM)-DWBA is usually employed. One first determines the optical potential by folding a Fermi distribution for the ground state matter density with a parameterized $\alpha$ nucleon interaction, determined by a fit to the elastic scattering cross-section. Next, the classical collective ISGMR and ISGMR2 transition densities of Eqs. (11) and (12) are folded with the $\alpha$ nucleon interaction to determine the transition potentials. Then the optical potential and the transition potentials are used as input for the DWBA code (see, e.g., Refs. [2,3]). Since only the energy-averaged ISM double transition density of Eq. (1) can be obtained within the PHDOM, we study the accuracy of this approach by calculating the excitation cross section within the Born approximation. In this approximation, the energy averaged transition strength function is proportional to the strength function $S_{V_{0, q}}$, corresponding to the external field $V_{0, q}(r)=\frac{\sin (q r)}{q r}$,

$$
\left\langle\sum_{s}\left|T_{\alpha^{\prime}, \alpha 0}\right|^{2} \delta\left(E_{s}-E_{0}-\omega\right)\right\rangle=\left|V_{\alpha A}(q)\right|^{2} \int V_{0, q}(r) \rho\left(r, r^{\prime}, \omega\right) V_{0, q}\left(r^{\prime}\right) d r d r^{\prime}
$$


Here, $V_{\alpha A}(q)$ is the Fourier transform of the $\alpha$-particle-nucleon interaction. In this work we calculate the isoscalar monopole excitation cross section at forward angle (at $0^{\circ}$ ). The value of the momentum transfer is given by $q=k_{\alpha}-k_{\alpha^{\prime}}$. We note that since the classical double transition densities for the ISGMR and ISGMR2 are factorized the cross-sections can be directly obtained from the transition strength function using the transition densities $\rho_{c, i}^{\Lambda}\left(r, r^{\prime}, \omega\right)$ of Eq. (13).

To check the possibility of the approximate factorization of the ISM double transition density $\rho\left(r, r^{\prime}, \omega\right) \approx \rho_{V_{0, i}}(r, \omega) \rho_{V_{0, i}}\left(r^{\prime}, \omega\right)$ in the energy regions of the ISGMR and the ISGMR2 we examine the quantity

$$
Q_{V_{0, i}}(\omega)=\frac{\left(\int \rho_{V_{0, i}}(r, \omega) V_{0, q}(r) d r\right)^{2}}{\int V_{0, q}(r) \rho\left(r, r^{\prime}, \omega\right) V_{0, q}\left(r^{\prime}\right) d r d r^{\prime}},
$$

where, the definition of Eqs. (14) and (15) is used for $\rho_{V_{0, i}}(r, \omega)$. For the same aim the microscopically corrected classical double transition densities of Eq. (13) can be also used:

$$
Q_{c, i}^{\Lambda}(\omega)=\frac{\int V_{0, q}(r) \rho_{c, i}^{\Lambda}\left(r, r^{\prime}, \omega\right) V_{0, q}\left(r^{\prime}\right) d r d r^{\prime}}{\int V_{0, q}(r) \rho\left(r, r^{\prime}, \omega\right) V_{0, q}\left(r^{\prime}\right) d r d r^{\prime}} .
$$

\section{INPUT QUANTITIES AND RESULTS OF CALCULATIONS}

The input quantities of the PHDOM calculations carried out in this work are the LandauMigdal $\mathrm{p}$-h interaction and a partially self-consistent phenomenological mean field together with the imaginary part of the effective optical-model potential. The parameters of the mean field consistent with the Landau-Migdal $\mathrm{p}$ - $\mathrm{h}$ interaction isovector part are described in details in Ref. [15] where the parameters of the model for ${ }^{208} \mathrm{~Pb}$ are also given. These parameters (see Table 1 of Ref. [15]) are obtained from the description of the observed neutron and proton single-quasiparticle spectra in ${ }^{208} \mathrm{~Pb}$. The values of $f^{e x}=-2.876$ and $f^{\text {in }}=0.0875$ for the strength parameters of the isoscalar part of the Landau-Migdal p-h interaction, $F(r)=C\left(f^{e x}-\left(f^{e x}-f^{i n}\right) f_{W S}(r)\right)$, where $C=300 \mathrm{MeV} \cdot \mathrm{fm}^{3}$, are determined from the condition that the isoscalar $1^{-}$spurious state, corresponding to the center-of-mass motion, should be at zero energy [16], and from the fit to the experimental value of the centroid energy of the ISGMR, $\omega_{1}=(13.96 \pm 0.20) \mathrm{MeV}$ [17]. The parameterization of the energy dependence of the imaginary part of the effective optical-model potential, $W(\omega)$, is given in Refs. [5,6,8]. The strength parameter $\alpha=0.07 \mathrm{MeV}^{-1}$ is found from the description within the model of the observed ISGMR $(F W H M)^{\exp }=(2.88 \pm 0.20) \mathrm{MeV}$ [17]. The strength of the real potential, $P(\omega)$, added to the mean field, is obtained from the imaginary part, $W(\omega)$, using the corresponding dispersive relationship [18]. We point out that all the model parameters have now been determined so that the main properties of any multipole isoscalar giant resonance in the ${ }^{208} \mathrm{~Pb}$ nucleus can be 
calculated without the need for additional parameters. This statement is naturally applied to the ISGMR2 as well.

In our analysis of properties of high-energy ISM excitations we first calculate the strength functions $S_{V_{0, i}}(\omega)$. In Figs. 1a and 1b we show the calculated fractions of the energyweighted strength functions $y_{i}(\omega)$, obtained within the PHDOM for the external fields $V_{0, i}$ of Eq. (5), which are associated with the ISGMR ( $\mathrm{i}=1)$ and the ISGMR2 (i=2), respectively. The parameter $\eta$ in the expression for $V_{0,2}$ is evaluated in accordance with the method described in Sect. 2, obtaining the value of $\eta=77 \mathrm{fm}^{2}$. To demonstrate the contribution of the Landau damping to formation of the considered giant resonances, we show in Figs. 1a and $1 \mathrm{~b}$ the fractions $y_{i}^{C R P A}(\omega)$ calculated within the continuum-RPA version, i.e. in the approximation $W(\omega)=P(\omega)=0$. As follows from the results presented in Fig. 1a, the ISGMR in such heavy nucleus as ${ }^{208} \mathrm{~Pb}$ exhibits, taking the spreading effect into account, a well-formed resonance. The centroid energy $\omega_{1}=13.8 \mathrm{MeV}$ and total width $\Gamma_{1}=2.9 \mathrm{MeV}$ calculated within our model for the excitation energy interval $10-35 \mathrm{MeV}$, are in agreement with the above-mentioned experimental quantities [17]. Nonetheless, due to the Landau damping the fraction $y_{1}(\omega)$ shown in Fig. 1a exhibits a gross structure: along with the main maximum there is a small peak at the energy $\approx 17 \mathrm{MeV}$. In contrast to the ISGMR, its overtone does not exhibit a well-formed resonance (Fig. 1b).

Considering the energy-averaged ISM double transition density, we show in Figs. 2-4, the calculated transition density $\rho\left(r, r^{\prime}, \omega\right)$ of Eq. (1) for $r^{\prime}=r$ at three energy points: in the vicinity of the maxima of the ISGMR and of the ISGMR2, and between these maxima. This transition density is compared with factorized projected and microscopically corrected classical double transition densities, $\rho_{i}(r, r, \omega)=\left(\rho_{i}(r, \omega)\right)^{2}$ and $\rho_{c, i}^{\Lambda}(r, r, \omega)=\left(\rho_{c, i}^{\Lambda}(r, \omega)\right)^{2}$, defined by Eqs. (14), (15) and (10)-(13), respectively. The comparison is given in Fig. 2 ( $\mathrm{i}=1)$ and Fig. 3 (i=2). It is clearly seen from Fig. 2, that only near the maximum of the ISGMR the quantities $\rho(r, r, \omega)$, $\rho_{i=1}(r, r, \omega)$ and $\rho_{c, i=1}^{\Lambda}(r, r, \omega)$ are close (especially near the nuclear surface). For other energies these quantities are quite different. As follows from Fig. 3, near the maximum of the ISGMR2 the quantities $\rho(r, r, \omega), \rho_{i=2}(r, r, \omega)$ and $\rho_{c, i=2}^{\Lambda}(r, r, \omega)$ are not so close. In Fig. 4 we plot results for $\mathrm{i}=1$ at a vicinity of the ISGMR. As expected, the difference between the corresponding microscopic and classical transition densities appears at the giant-resonance tails.

In Figure 5, we plot the quantity $\rho\left(r, r^{\prime}, \omega\right)$ as a function of $r$ for the fixed value case of $r^{\prime}$ and excitation energy $\omega$ in a vicinity of the ISGMR and ISGMR2, to trace the radialdependence change from the one-node to two-node dependence.

We compare the projected and microscopically corrected classical transition densities, $\rho_{i}(r, \omega)$ and $\rho_{c, i}^{\Lambda}(r, \omega)$, at a vicinity of the ISGMR (i=1) (Fig. 6) and its overtone (i=2) (Fig. 7). As expected, the difference between the corresponding microscopic and classical transition densities is larger at the giant-resonance tails.

Further, we consider the energy-averaged strength function $S_{V_{0, q}}(\omega)$ of Eq. (19), which determines in the Born approximation the excitation cross-section of the ISGMR and its overtone 
in ${ }^{208} \mathrm{~Pb}$ by $240 \mathrm{MeV} \alpha$-particle scattering [2] ( $E_{\alpha}=\frac{\mu}{m_{\alpha}} 240 \mathrm{MeV}, m_{\alpha}$ is the $\alpha$-particle mass). To avoid an appearance of small negative values of the strength function $S_{V_{0, q}}(\omega)$, we take the corresponding external field as $V_{0, q}(r)=\frac{\sin (q r)}{q r}-1$. In such a way we restore a weak violation of the model unitarity [14]. It is natural that the strength function $S_{V_{0, q}}(\omega)$ (Figs. 8 and 9) exhibits the maxima, corresponding to the ISGMR and its overtone. In Figs. 8 and 9 we show the strength function evaluated via energy-averaged microscopic double transition density $\rho\left(r, r^{\prime}, \omega\right)$ in comparison with the strength functions evaluated with the use of the factorized projected and microscopically corrected classical transition densities, $\rho_{i}(r, \omega)$ and $\rho_{c, i}^{\Lambda}(r, \omega)$. As follows from this comparison, the description with the use of the factorized projected transition densities reproduces satisfactorily the "exact" description in a vicinity of the ISGMR ( $\mathrm{i}=1)$ and ISGMR2 $(\mathrm{i}=2)$.

The quality of the approximate descriptions of the strength function $S_{V_{0, q}}(\omega)$ (Figs. 8 and 9) illustrates the ratios $Q_{V_{0}, i}(\omega)$ of Eq. (20) and $Q_{c, i}^{\Lambda}(\omega)$ of Eq. (21) shown in Figs. $10(\mathrm{i}=1)$ and 11 ( $\mathrm{i}=2)$, respectively. The solid thick lines of Figs. 10 and 11 are obtained from the projected one body densities using the corresponding scattering operators of Eq. (5) for the ISGMR and ISGMR2. The corresponding solid thin lines were obtained using the semiclassical transition densities of Eqs. (11) and (12), respectively. As follows from consideration of these ratios, the approximate description of $S_{V_{0}, q}(\omega)$ in terms of the factorized projected double transition densities is preferable (obtaining a value of 1), as compared with the description in terms of the microscopically corrected classical double transition densities.

\section{CONCLUDING REMARKS}

We have presented in this work results of the first application of the recently developed particle-hole dispersive optical model (PHDOM) to calculate the energy-averaged double transition density for the isoscalar monopole excitations in ${ }^{208} \mathrm{~Pb}$ over a wide range energy region, which includes the energy regions of the ISGMR and its overtone (ISGMR2). The corresponding energy-averaged strength functions were also analyzed. The calculated double transition density was studied as functions of the excitation energy and position. The results were compared with the double transition densities constructed on projected and classical collective model transition densities for the ISGMR and ISGMR2. We have demonstrated, in particular, that in the intermediate excitation energy region (between the energies of the ISGMR and ISGMR2) the double transition density is quite different from that obtained from the classical transition densities, which are commonly used in the experimental analysis of hadron inelastic scattering cross sections for the ISM excitations. As a first step towards implications of the obtained results on the experimental analysis of hadron excitation cross sections, we have demonstrated possibilities of the model in employing the microscopic double and projected single transition densities for the description, within the Born approximation, of the inelastic $\alpha$ - 
scattering at zero angle cross sections for ISM excitations. Considering as a probe the Born approximation, we show that the use of the projected transition densities is preferable (Figs. 8 and 9). Of course, a more realistic reaction calculation using the distorted wave Born approximation should be carried out. We note that the input to commonly used codes are the optical potential and the one body transition potential. As a first step, one can use in these codes the microscopic projected transition densities within a rather large energy interval in the vicinity of the ISGMR and ISGMR2 (Figs. 10 and 11). The new codes should be designed to admit as input the double transition density. Such a study is planned to be continued.

\section{ACKNOWLEGEMENTS}

This work was supported by the Russian Foundation for Basic Research under grant No. 15-02-08007-a (M.L.G., B.A.T., M.H.U.), by the US Department of Energy under grant No. DE-FG02-93ER40773 (S.S.), and the Competitiveness Program of National Research Nuclear University «MEPhI» (M.H.U.). S.S is very grateful to the nice hospitality of the NRNU «MEPhI» during his visit and acknowledges the support by the Ministry of Education and Science of the Russian Federation for the Competitiveness Program of the NRNU «MEPhI», under grant No. 02.a03.21.0005. M.L.G. is very grateful to the Cyclotron Institute, Texas A\&M University, for the support and nice hospitality provided to him during his visit. 


\section{REFERENCES}

[1] J. P. Blaizot, Phys. Rept. 64 (1980) 171.

[2] D. H. Youngblood, Y.-W. Lui, Krishicayan, J. Button, M. R. Anders, M. L. Gorelik, M. H. Urin, and S. Shlomo, Phys. Rev. C 88 (2013) 021301(R).

[3] A. Kolomiets, O. Pochivalov, and S. Shlomo, Phys. Rev. C 61 (2000) 034312.

[4] A. B. Migdal, Theory of Finite Fermi Systems and Applications to Atomic Nuclei, Interscience, New York, 1967.

[5] M. H. Urin, Phys. At. Nucl. 74 (2011) 1189.

[6] M. H. Urin, Phys. Rev. C 87 (2013) 044330.

[7] S. Shlomo and G. Bertsch, Nucl. Phys. A243 (1975) 507.

[8] B. A. Tulupov and M. H. Urin, Phys. Rev. C 90 (2014) 034613.

[9] M. L. Gorelik, S. Shlomo, B. A. Tulupov and M. H. Urin, Phys. At. Nucl. 78 (2015) 595.

[10] M. L. Gorelik and M. H. Urin, Phys. Rev. C 64 (2001) 047301.

[11] M. L. Gorelik, I. V. Safonov, and M. H. Urin, Phys. Rev. C 69 (2004) 054322.

[12] M. H. Urin, Nucl. Phys. A811 (2008) 107.

[13] S. Shlomo, V. M. Kolomietz, and B. K. Agrawal, Phys. Rev. C 68 (2003) 064301.

[14] M. L. Gorelik, S. Shlomo, B. A. Tulupov and M. H. Urin, EPJ Web of Conferences 107 (2016) 05008.

[15] G. V. Kolomiytsev, S. Yu. Igashov, M. H. Urin, Phys. At. Nucl. 77 (2014) 1105.

[16] M. L. Gorelik, S. Shlomo, and M. H. Urin, Phys. Rev. C 62 (2000) 044301.

[17] D. H. Youngblood, Y.-W. Lui, H. L. Clark, et al., Phys. Rev. C 69 (2004) 034315.

[18] B. A. Tulupov, M. H. Urin, Phys. At. Nucl. 72 (2009) 737. 


\section{FIGURE CAPTIONS:}

Fig. 1. Fractions of the energy-weighted strength functions $y_{i}(\omega)$ (the solid thick line) in comparison with $y_{i}^{c R P A}(\omega)$ (the solid thin line) for the ISGMR (i=1) and ISGMR2 (i=2) (Figs. 1a and $1 \mathrm{~b}$, respectively).

Fig. 2. The ISM double transition density $\rho(r, r, \omega)$ calculated at the different excitation energies: 13.8 MeV (a), $23 \mathrm{MeV}$ (b), $33 \mathrm{MeV}$ (c) (the solid thick lines) in a comparison with the factorized projected and microscopically corrected classical double transition densities, $\rho_{i}(r, r, \omega)$ and $\rho_{c, i}^{\Lambda}(r, r, \omega)$ (the solid thin and dashed lines, respectively), for $\mathrm{i}=1$.

Fig. 3. Same as Fig. 2 but for $i=2$.

Fig. 4. Same as Fig. 2 for $i=1$ but at the different excitation energies in a vicinity of the ISGMR: 10.8 MeV (a), 13.8 MeV (b), $16.8 \mathrm{MeV}$ (c).

Fig. 5. The ISM double transition density $\rho\left(r, r^{\prime}, \omega\right)$ calculated at the different excitation energies: $13.8 \mathrm{MeV}$ (a), $23 \mathrm{MeV}$ (b), $33 \mathrm{MeV}$ (c) for the several fixed quantities $r^{\prime}=5 \mathrm{fm}$, $r^{\prime}=7 \mathrm{fm}$ and $r^{\prime}=9 \mathrm{fm} \mathrm{fm}$ (the solid thick, solid thin and dashed lines, respectively).

Fig. 6. A comparison of the projected and microscopically corrected classical single transition densities, $\rho_{i}(r, \omega)$ (the solid thick lines) and $\rho_{c, i}^{\Lambda}(r, \omega)$ (the solid thin lines). The comparison is given for the excitation energies: $10.8 \mathrm{MeV}$ (a), 13.8 MeV (b), 16.8 MeV (c) in a vicinity of the ISGMR (i=1).

Fig. 7. Same as Fig. 6 but for the excitation energies: $28 \mathrm{MeV}$ (a), $33 \mathrm{MeV}$ (b), $38 \mathrm{MeV}$ (c) in a vicinity of the ISGMR2 main peak $(i=2)$.

Fig. 8. The strength function $S_{V_{0, q}}(\omega)$ shown (the solid thick line) in a comparison with the strength function, corresponding to the same external field but calculated with the use of the projected double transition densities $\rho_{i}\left(r, r^{\prime}, \omega\right)$ (the solid thin line) and microscopically corrected classical double transition densities $\rho_{c, i}^{\Lambda}\left(r, r^{\prime}, \omega\right)$ (the dashed line) for the ISGMR (i=1). The corresponding $q$ values are in the interval $0.07-0.52 \mathrm{fm}^{-1}$.

Fig. 9. Same as Fig. 8 but for the ISGMR2 (i=2) and the $q$ values are in the interval $0.04-1.25 \mathrm{fm}^{-1}$.

Fig. 10. A comparison of the ratios of $Q_{V_{0}, i}(\omega)$ of Eq. (20) (the solid thick line) and $Q_{c, i}^{\Lambda}(\omega)$ of Eq. (21) (the solid thin line) for the ISGMR (i=1).

Fig. 11. Same as Fig. 10 but for the ISGMR2 (i=2). 

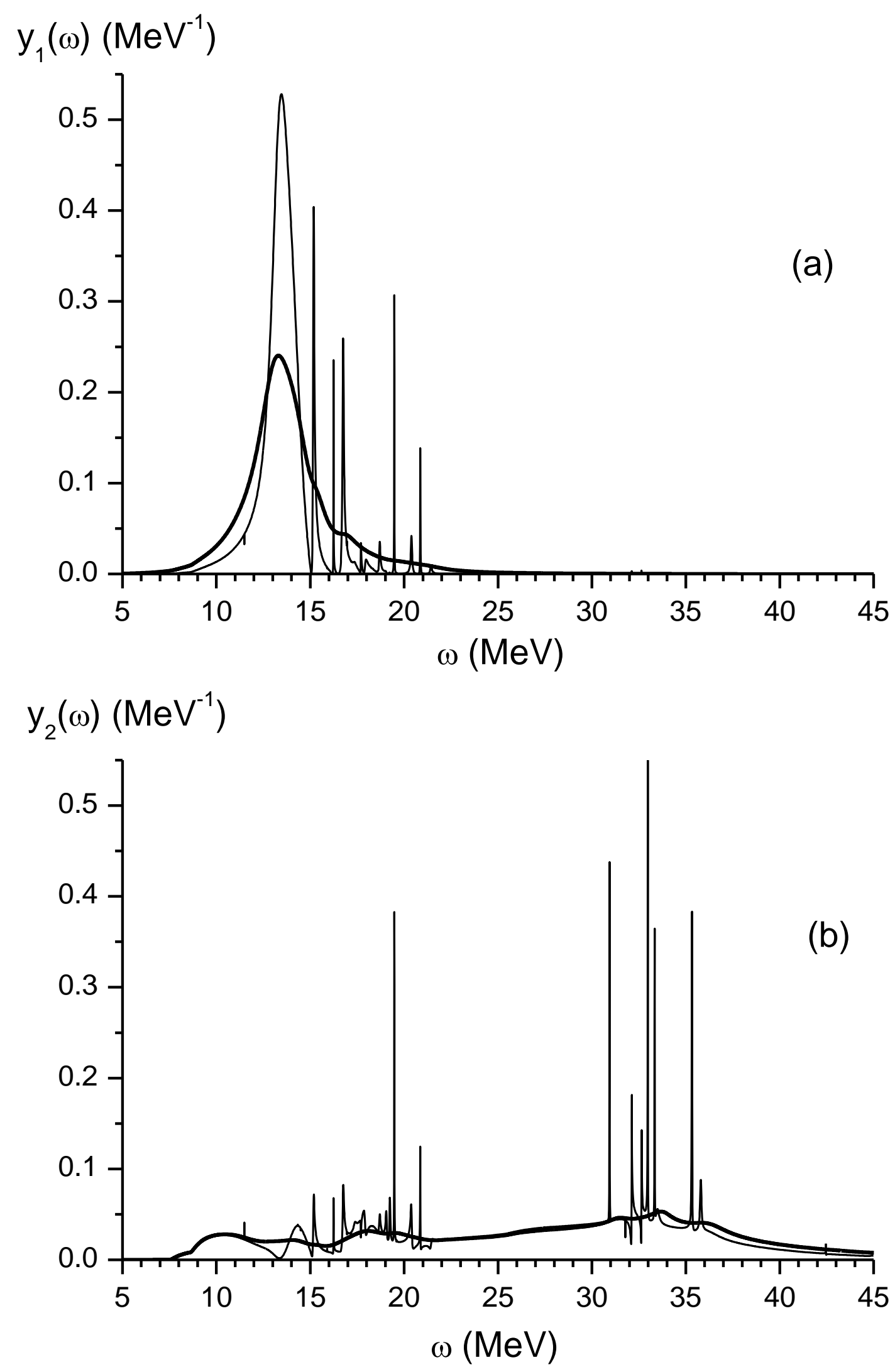

Fig. 1 


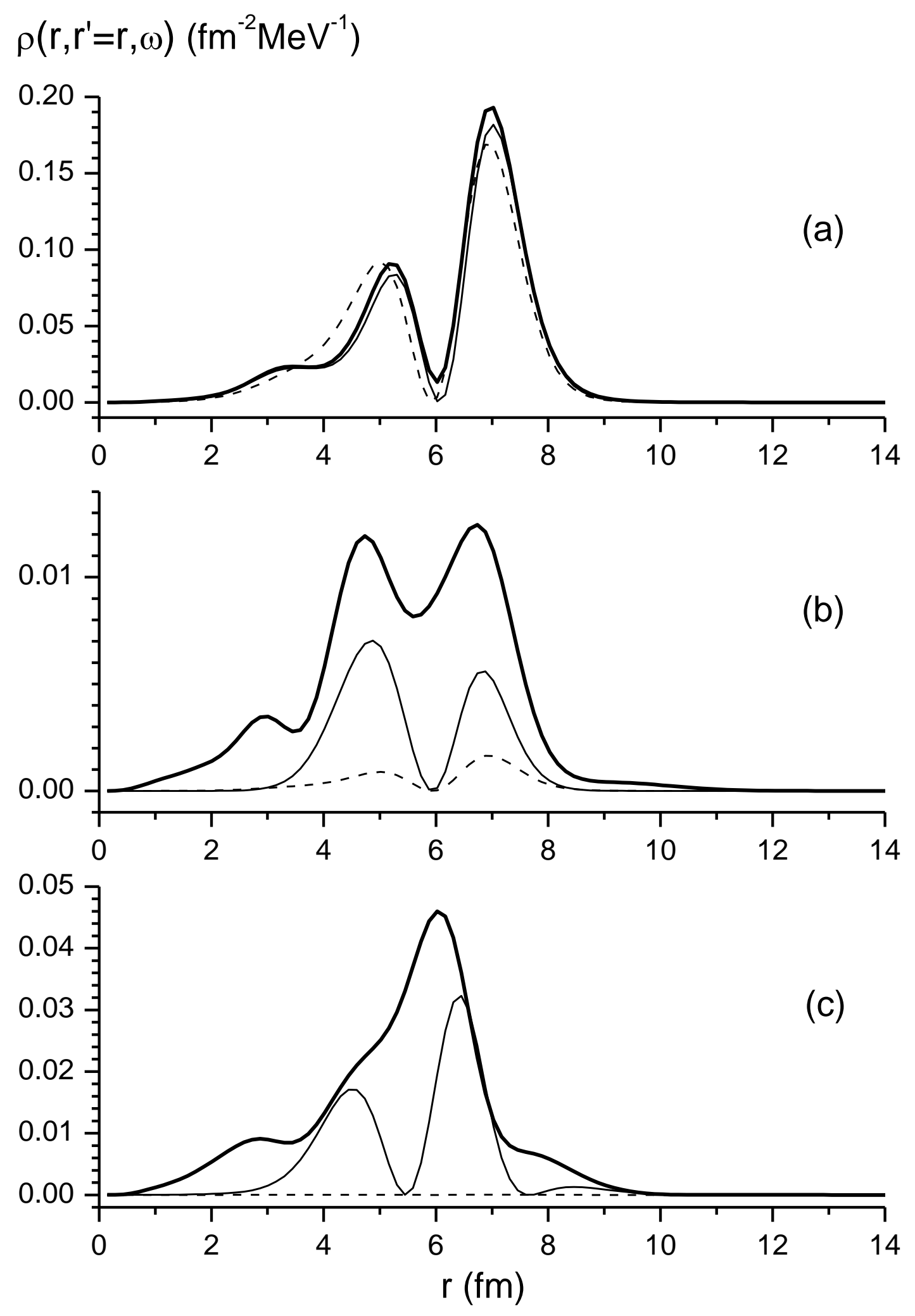

Fig. 2 


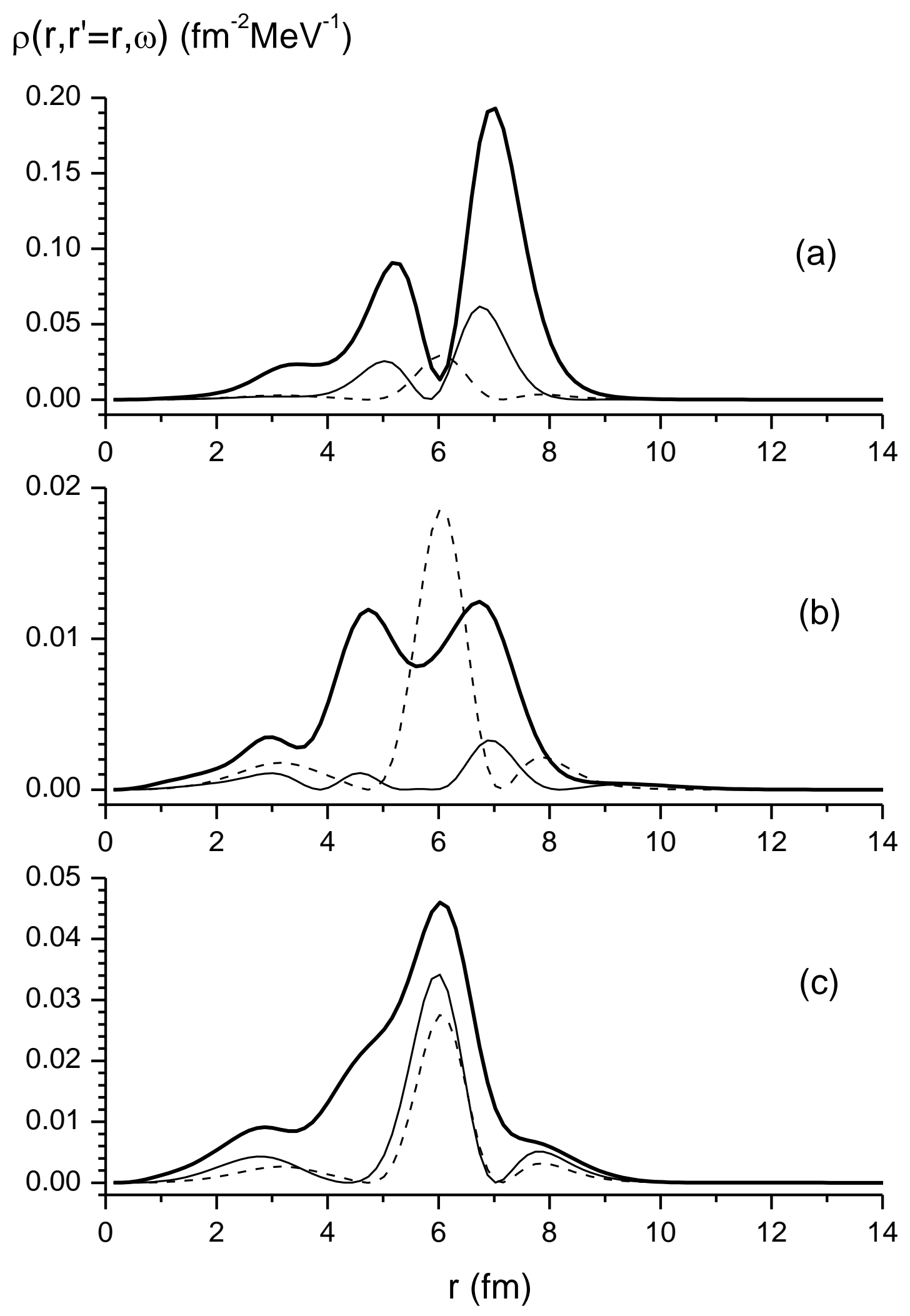

Fig. 3 

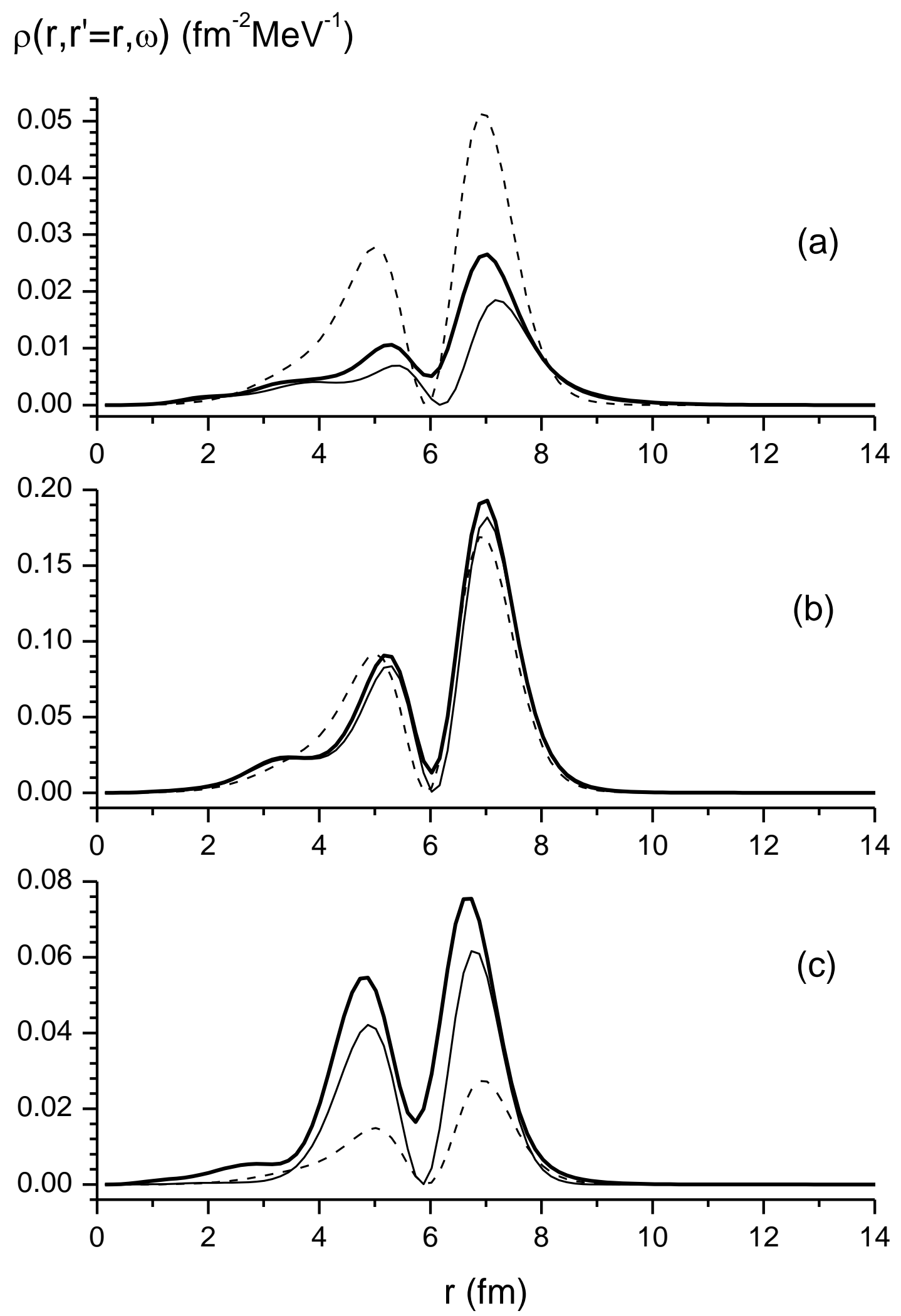

Fig. 4 

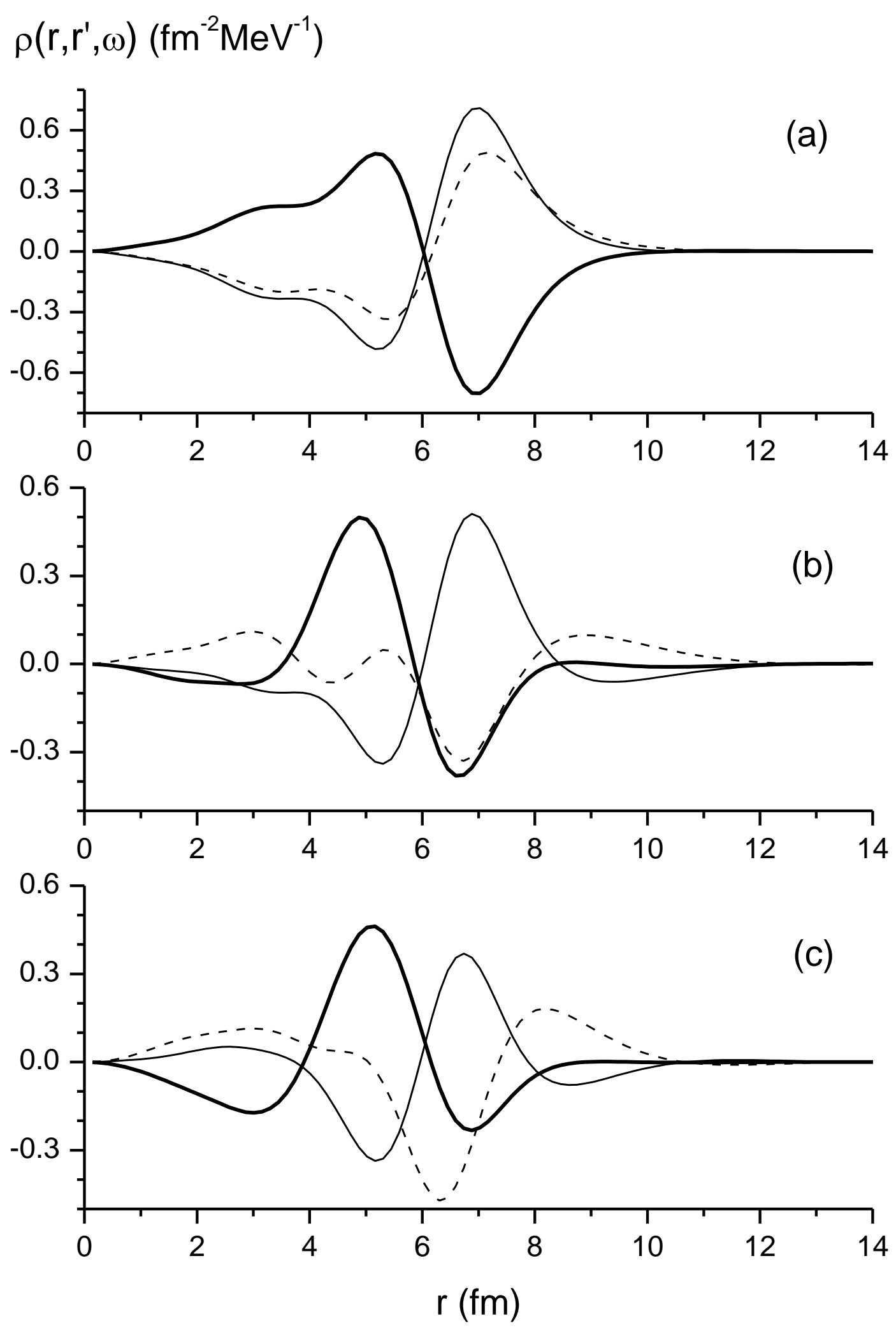

Fig. 5 

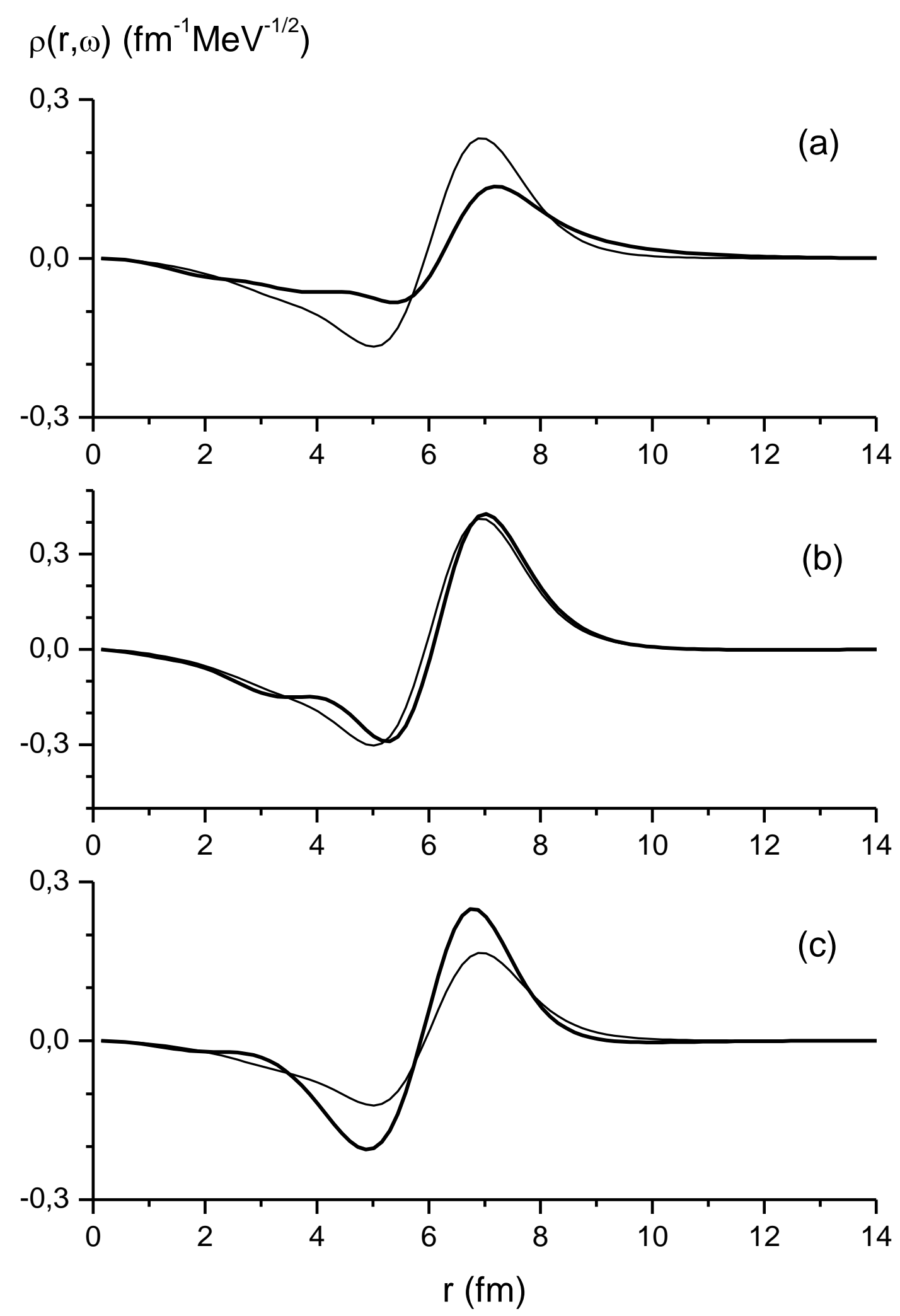

Fig. 6 


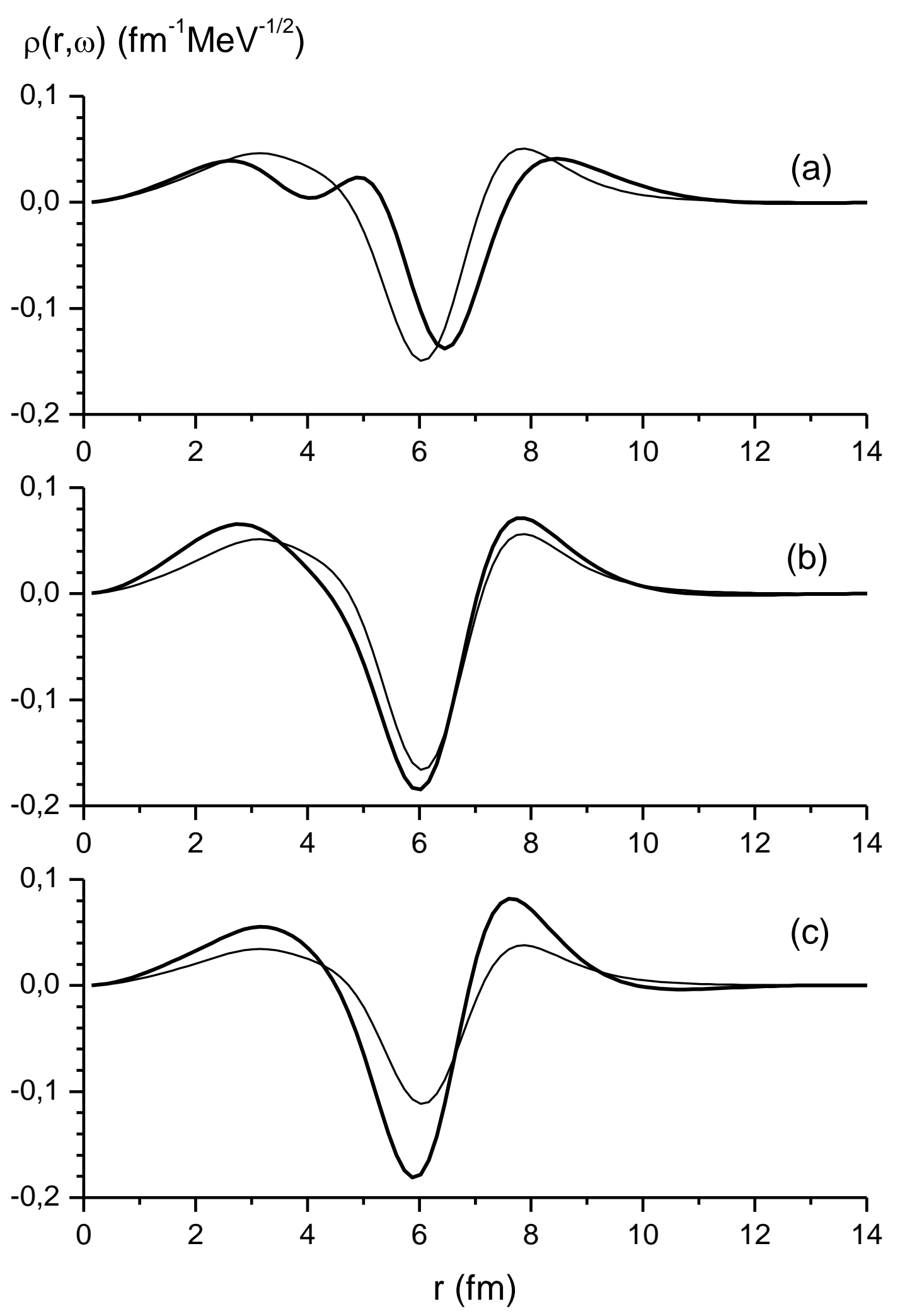

Fig. 7 


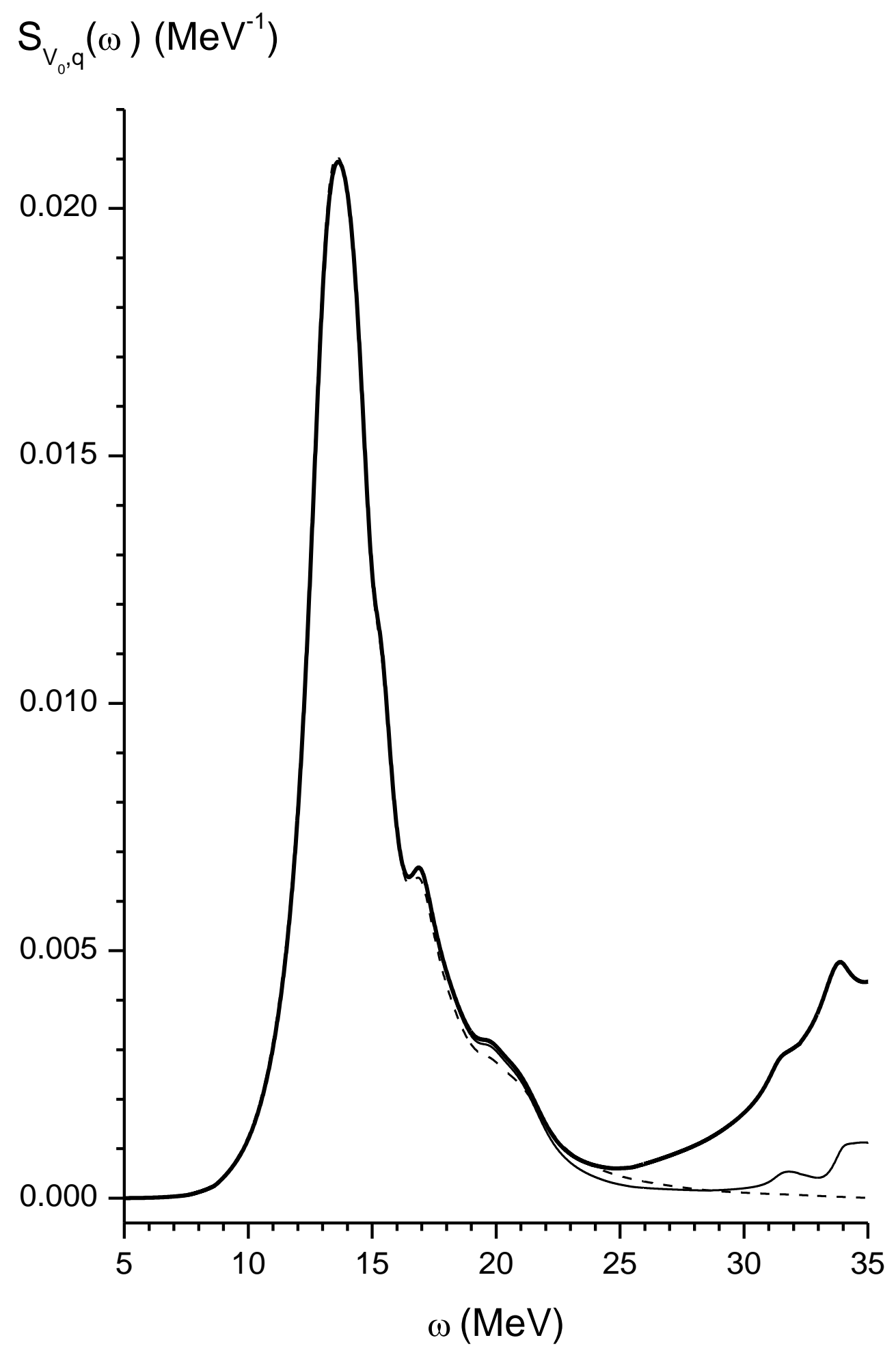

Fig. 8 


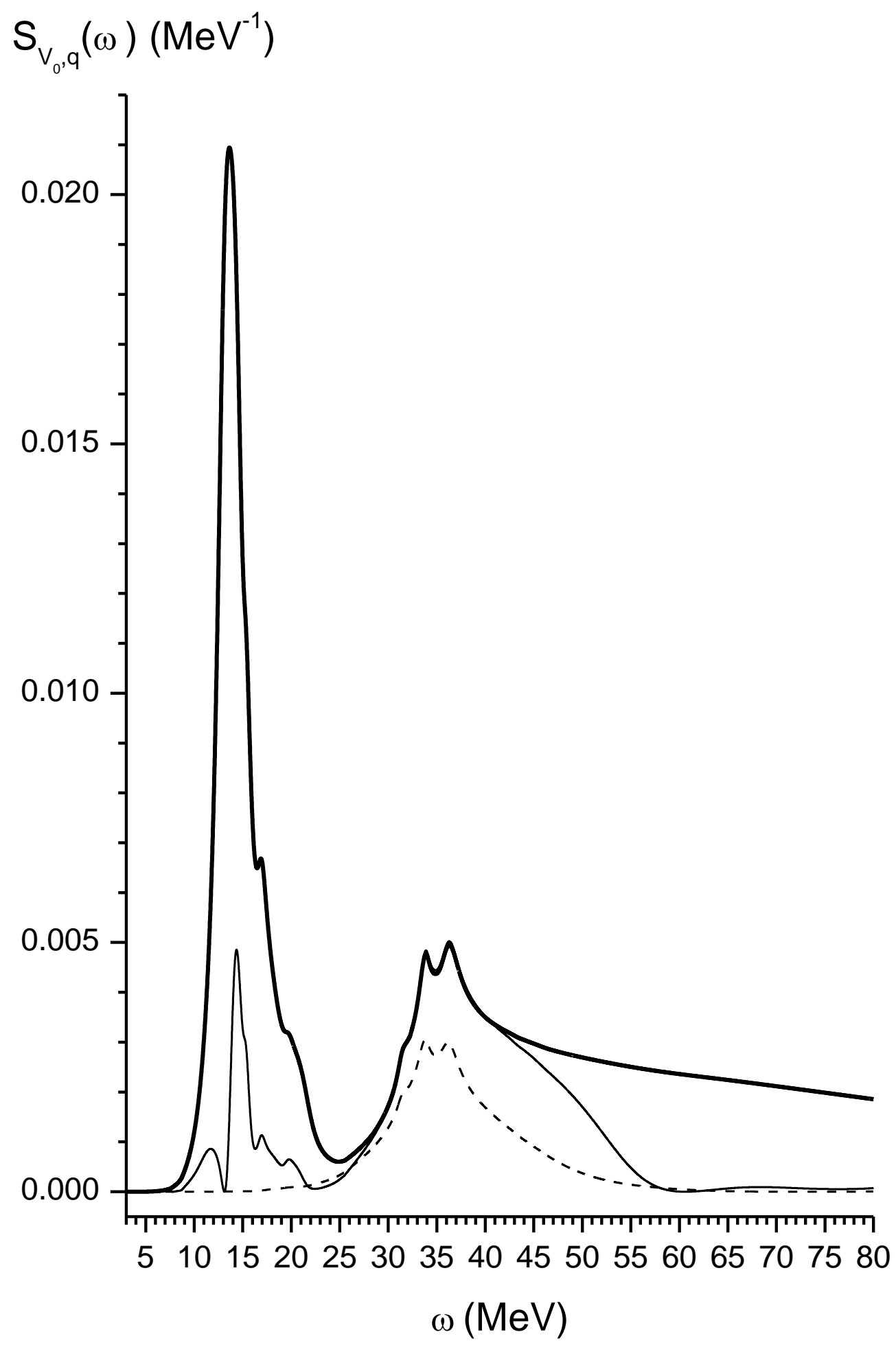

Fig. 9 


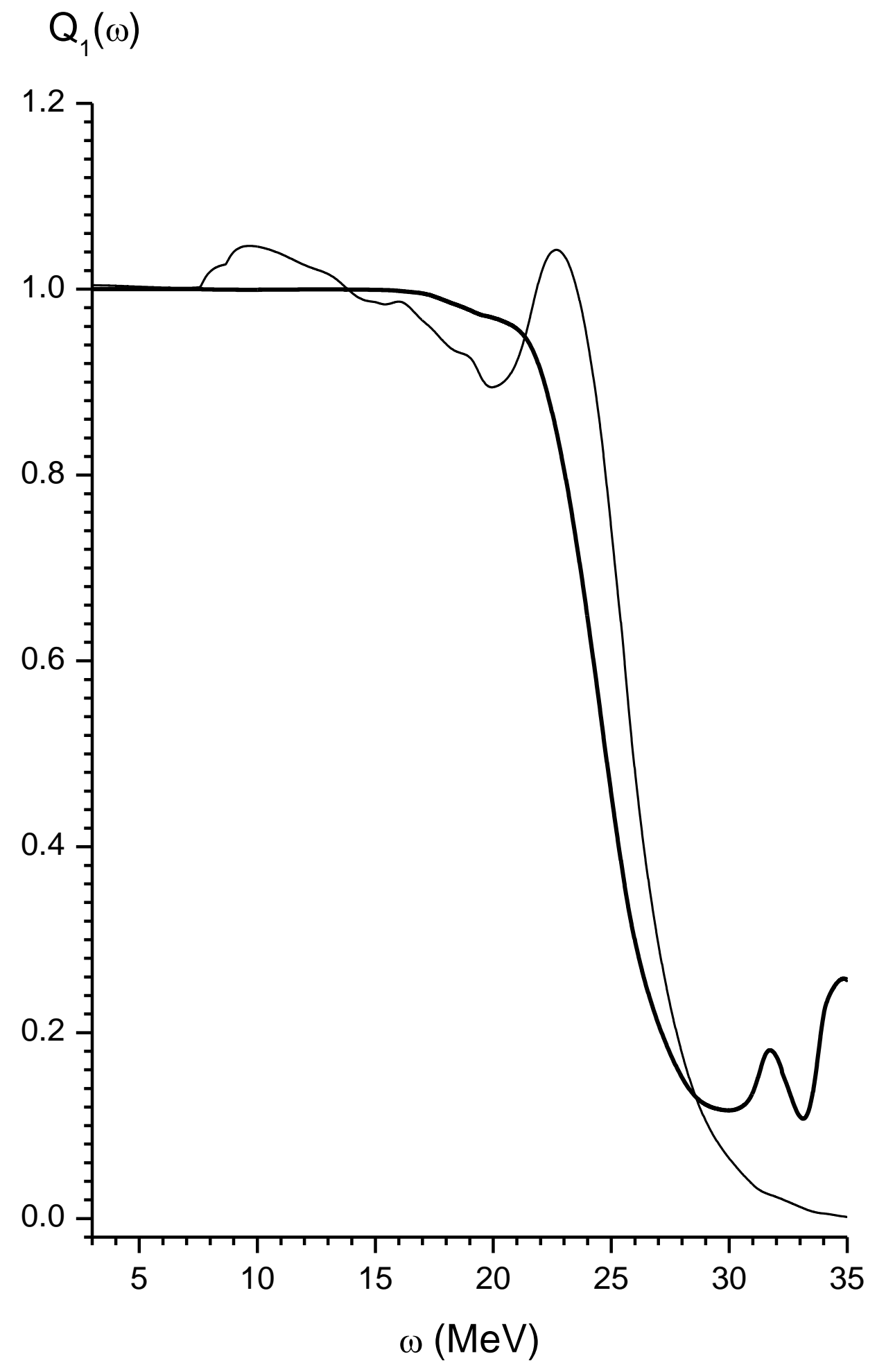

Fig. 10 


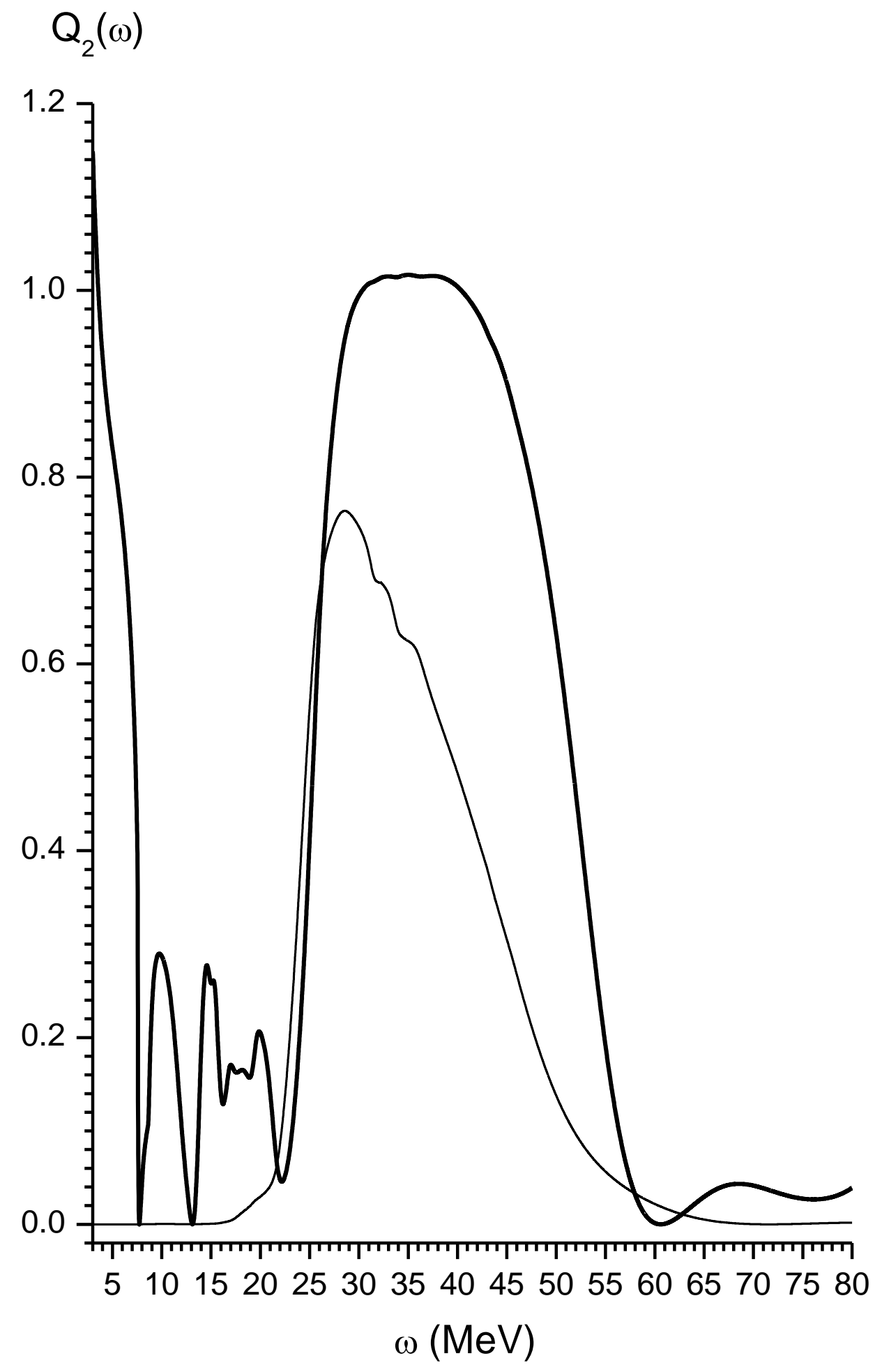

Fig. 11 